\title{
A Robust Method Applied to Human Detection
}

\author{
Seyyed Meysam Hosseini, Hasan Farsi, Member, IACSIT
}

\begin{abstract}
PC-SVM is a new developed support vector machine classifier with probabilistic constrains which presence of samples probability in each class is determined based on a distribution function. The presence of noise causes incorrect calculation of support vectors thereupon margin can not be maximized. In the Pc-SVM, constraints boundaries and constraints occurrence have probability density functions which it helps for achieving maximum margin. The main target of this paper is introducing a robust visual object recognition based on PC- SVM. Human detection is used as benchmark problem for the proposed algorithm. Experimental results show superiority of the probabilistic constraints support vector machine (PC-SVM) relative to standard SVM in human detection.
\end{abstract}

Index Terms - pc-svm, human detection, histograms of oriented gradients.

\section{INTRODUCTION}

So far, a lot of works have been done around the object detection. Papageorgiou [1] describe a pedestrian detector based on a polynomial SVM using rectified Haar wavelets as input descriptors, with sub windows based variant in [2].In [11], body parts were represented by combinations of joint orientation and position histograms, Separate Adaboost detectors were trained for several body parts. Ronfard etal [7] build an articulated body detector by incorporating SVM based limb classifiers over 1st and 2nd order Gaussian filters in a dynamic programming framework similar to those of Felzenszwalb \& Huttenlocher [8] and Ioffe \& Forsyth [9]. Mikolajczyk [10] use combinations of orientation position histograms with binary-threshold gradient magnitudes to build a parts based method containing detectors for faces, heads, and front and side profiles of upper and lower body parts. In contrast, the detector in this paper uses a simpler architecture with a single detection window, but appears to give significantly higher performance on pedestrian images. Detecting humans in images is a challenging task owing to their variable appearance, Occlusions, unconstrained illumination and Wide variety of articulated poses that they can adopt. A robust feature set that allows the human form to be distinct cleanly even in complex backgrounds under difficult illumination is necessary. Hence in this paper we study the issue of human detection base on PC-SVM by using locally Normalized Histogram of Oriented Gradient (HOG) descriptors that provide excellent performance relative to other existing feature sets including wavelets $[2,6]$.The paper is organized as follows. First, we mention the proposal algorithm briefly in Section 2. Section 3 is appropriated to the SVM and PC-SVM foundation respectively, finally collected HOG is used for adopting PC-SVM, which is followed by experimental result over human/non-human detection in Section 4 , and consequently we conclude with a number of comments in Section 5.

\section{Proposal Algorithm}

This section gives a brief overview of our feature extraction and PC-SVM adopting chain, which is summarized in fig. 1. The method is based on PC-SVM adopting by estimating well-normalized local histograms of image gradient orientations in a dense grid. HOG feature extraction block for better result may be dividing into several sub blocks as Gamma/Colour Normalization, Orientation cells, Block Normalization, Computing histogram of gradient orientations. Proposal algorithm is implemented by dividing the image window into small cells, for each cell accumulating a local 1-D histogram of gradient directions or edge orientations over the pixels of the cell. The combined histogram entries form the representation. For better invariance to illumination, shadowing, etc., it is also useful to contrast-normalize the local responses before using them [14]. This can be done by accumulating a measure of local histogram over somewhat larger spatial regions and using the results to normalize all of the cells in the block. We will refer to the normalized descriptor blocks as Histogram of Oriented Gradient (HOG) descriptors. Tiling the detection window with a dense (in fact, overlapping) grid of HOG descriptors and using the combined feature vector in a PC-SVM based window classifier gives our human detection chain (fig. 1).In [14],it is shown that the overall HOG feature vector (at level $\mathrm{L})$ for a cell $(\mathrm{Cx}, \mathrm{Cy})$ is as fellows :

$$
\begin{aligned}
& \operatorname{HOG}_{\text {cell }}^{l}(c x, c y)= \\
& \frac{[f(c x-1, c y-1)|f(c x, c y-1)| f(c x-1, c y) \mid f(c x, c y)]}{E(c x-1, c y-1)+E(c x, c y-1)+E(c x-1, c y)+E(c x, c y)}
\end{aligned}
$$

Where $\mathrm{f}(\mathrm{Cx}, \mathrm{Cy}), \mathrm{E}(\mathrm{Cx}, \mathrm{Cy})$ indicate the weighted by the magnitude of the gradient at that pixel and energy of a cell, respectively. Note that, $\mid$ denotes concatenation of features.

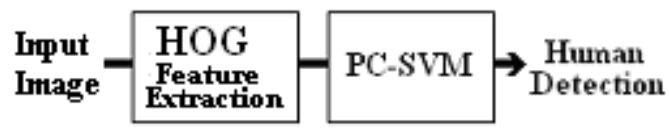

Fig1: proposal algorithm 


\section{Clasiffication By PC-SvM}

PC-SVM to be developed version of SVM that commonly used for classification, but in this paper best classification needed ,because feature (HOG) for human and non-human have overlap area that make badly accuracy in human object classification, in continue firstly we describe the fundamental of last SVM and then describe the probabilistic constraint for best developing.

\section{A. SVM foundation}

The support vector machine is a linear machine, It is not possible for such a set of training data to construct a separating hyperplane without encountering classification error. In next stage expressed new constraint base SVM version that able to constructing a separating hyperplane without encountering classification error.

\section{B. Optimal hyperplane}

In This section we are considering the optimal hyperplane for SVM that able to minimize the classification errors. Let $\mathrm{s}=\left\{\left(x_{i}, y_{i}\right)\right\}_{i=1}^{n}$ be a set of $n$ training samples, where $x_{i} \in R^{m}$. is an $m$-dimensional sample in the input space, and $d_{i} \in\{-1,1\}$ is the class label of $x_{i}$. Let $w_{0}$ and $b_{0}$ denote the optimum values of the weight vector and bias respectively. The hyperplane can be represented as:

$$
w_{0}^{T} x+b_{0}=0
$$

Where $w=\left[w_{1}, w_{2}, \ldots, w_{m}\right]^{T}$ and $x=\left[x_{1}, x_{2}, \ldots, x_{m}\right]^{T}$ $w$ is the normal vector of the hyperplane, and $b$ is the bias that is a scalar. Optimal hyperplane can be obtained by solving the following optimization problem:

$$
\begin{gathered}
\frac{1}{2}\|W\|^{2}+C \sum_{i=1}^{n} \xi_{i} \\
P R\left(d_{i}\left(w^{T} x_{i}+b\right) \geq u_{i}-\xi_{i}\right) \geq \delta_{i}, \\
i=1, \ldots, n \quad \xi_{i} \geq 0,
\end{gathered}
$$

Where $u_{i}$ are independent random variables with known distribution functions and $0 \leq \delta_{i} \leq 1$ is value of effect of $i^{\text {th }}$ samples in the position of optimal hyperplane or selection of support vectors. Then (3) can be written as,

$$
d_{i}\left(w^{T} x_{i}+b\right) \geq F_{i}^{-1}\left(\beta_{i}\right)
$$

Where $\beta_{\mathrm{i}}=1-\delta_{\mathrm{i}}$, and $F_{i}^{-1}($.$) is the inverse distribution$ function of the variable $u_{i}-\xi_{i}$, with $i=1, \ldots, n$; which has to be continuous. Similar to the conventional SVM, the optimization problem PC-SVM can be transformed into its dual problem. One of reason for moving to the dual form of the problem is that of, constraints in the dual form of the problem are significantly simpler than those in the primal form and other subject is that of in the dual form, the training data will appear only in the form of dot products

$$
F^{-1}\left(\beta_{i}\right)-d_{i}\left(w x_{i}+b\right) \leq 0
$$

Optimization procedure continues as follows,

$$
\begin{gathered}
J\left(w, b, \alpha, \xi_{i}\right)= \\
\frac{1}{2} w^{T} w+c \sum_{i=1}^{n} \xi_{i}+\sum_{i=1}^{n} \alpha_{i}\left[F^{-1}\left(\beta_{i}\right)-d_{i}\left(w x_{i}+b\right)\right] \\
\frac{\partial J}{\partial w}=w-\sum_{i=1}^{n} \alpha_{i} d_{i} x_{i}=0 \\
\frac{\partial J}{\partial b}=\sum_{i=1}^{n} \alpha_{i} d_{i}=0 \\
\frac{\partial J}{\partial \xi_{i}}=c=0 \quad i=1, \ldots, n
\end{gathered}
$$

For solving this problem, it is converted to dual form. With given the training sample $\{(\mathrm{xi}, \mathrm{di})\}_{i=1}^{n}$ find the Lagrange multipliers $\{\alpha\}_{i=1}^{n}$ that maximize the objective function.

$$
\begin{gathered}
\operatorname{Maximize} \sum_{i=1}^{n} \alpha_{i} F^{-1}\left(\beta_{i}\right)-\frac{1}{2} \sum_{i=1}^{n} \sum_{j=1}^{n} \alpha_{i} \alpha_{j} d_{i} d_{j} x_{i} x_{j} \\
\sum_{i=1}^{n} d_{i} \alpha_{i}=0 \quad \text { s.t. } \\
i=1, \ldots, n \leq \alpha_{i} 0
\end{gathered}
$$

Sadoghi in [] get the following properties that be derived from the Kuan-Tucker conditions of optimization theory.

$$
\begin{aligned}
& \left.\begin{array}{c}
\sum_{i=1}^{n} \alpha_{i} d_{i}=0 \\
0 \leq \alpha_{i}
\end{array}\right\} \\
& F^{-1}\left(\beta_{i}\right)-d_{i}\left(w x_{i}+b\right) \leq 0 \quad i=1, \ldots, n \\
& \alpha_{i}\left[F^{-1}\left(\beta_{i}\right)-d_{i}\left(w x_{i}+b\right)\right] \leq 0 \quad i=1, \ldots, n
\end{aligned}
$$

Sadoghi used the optimum Lagrange multipliers, that denoted by $\alpha_{0 i}$, and it is possible to compute the optimum weight vector $w_{0}$ using

$$
\begin{gathered}
w_{0}=\sum_{i=1}^{n} \alpha_{i} d_{i} x_{i} \\
b=\left\{\begin{array}{l}
F^{-1}\left(\beta_{i}\right)-w x_{i} \\
-F^{-1}\left(\beta_{i}\right)-w x_{i}
\end{array}\right. \\
i=1, \ldots, n \quad \xi_{i}=1-d_{i}\left(w^{T} x_{i}+b\right)
\end{gathered}
$$

But in practical works this type of calculating be hard, hence we are used the PSO algorithm as described in [] to computing the optimum weight vector. in this way we get goodly estimated optimum weight vector and we are used it to detect human at this work.

In previous works soft-margin linear SVM frequently used by especial penalty deal that they pay in allowing slack variables in a soft-margin SVM. But in last SVM base works noise is caused incorrect calculation of support vectors thereupon margin can not be maximized, hence in this work we use new developed SVM version that able to maximize it. 
Several human example images and several non-human example images were used to generate the training set. The cell size was chosen by following [14] as $8 \times 8$. Each image was of size $32 \times 96$ and only a single level of resolution $(\mathrm{L}=1)$ was used to generate the training examples. Thus, each image generated 33 cell features. Overall, there were 825 human vectors and 825 non-human training vectors. 800 vectors were randomly selected from each set and then given for training to the PC-SVM classifier.

\section{Simulation Result}

New SVM classifier adopting as expressed lastly by human and non-human images and classify this tow classes by using feature set of HOG as depicted in the fig. 2 and 3.Testing proposal algorithm in compare with last work that be base SVM give clearly result (table.1), this result illustrate the superiority of proposal algorithm.

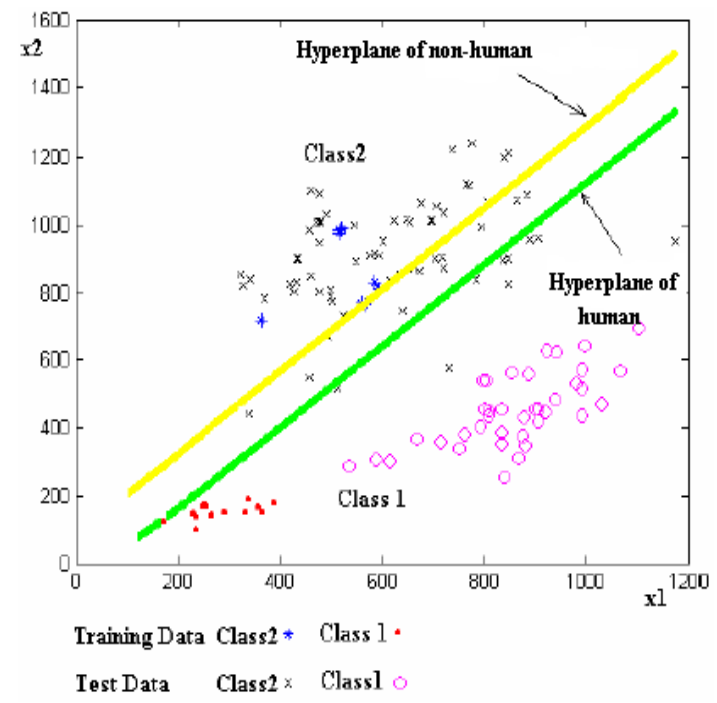

Fig.2: Training and testing data's in feature space $(2 \mathrm{x}-1 \mathrm{x})$. Hyperplanes are obtained using standard SVM but in the PC-SVM hyperplane of class 1 moves to center of class 1 and result will be higher recognition rate.
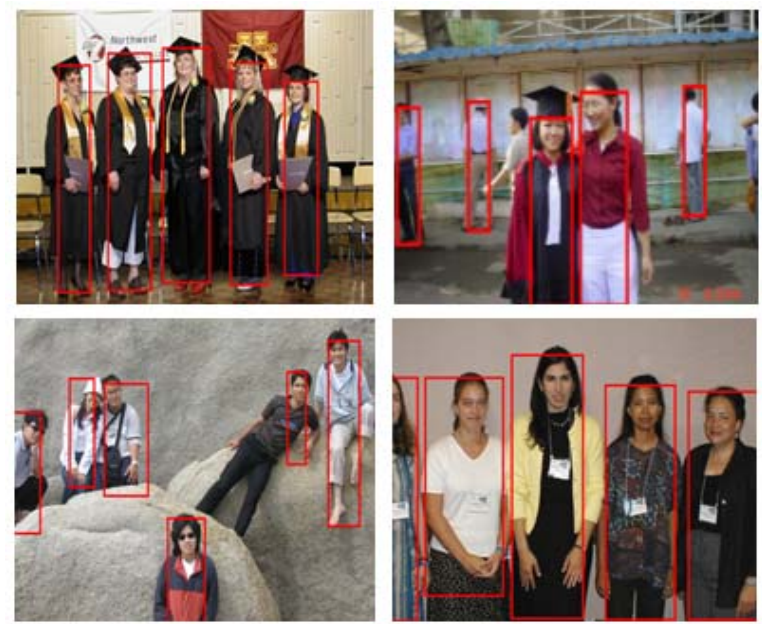

Fig3: proposal algorithm result

TABLE 1: RESULT OF SVM AND PC-SVM COMPARISON
PC-SVM Result

\begin{tabular}{|c|c|c|}
\hline Class name & $\begin{array}{c}\text { Class 1 as for } \\
\text { number } \mathbf{2}\end{array}$ & $\begin{array}{c}\text { Class 2 as for } \\
\text { number } \mathbf{5}\end{array}$ \\
\hline Class 1 as for number 2 & 100 & 0 \\
\hline Class 2 as for number 5 & 14.10 & 85.90 \\
\hline \multicolumn{3}{|c|}{ SvM Result } \\
\hline Class name & $\begin{array}{c}\text { Class 1 as for } \\
\text { number } \mathbf{2}\end{array}$ & $\begin{array}{c}\text { Class 2 as for } \\
\text { number } \mathbf{5}\end{array}$ \\
\hline Class 1 as for number 2 & 100 & 0 \\
\hline Class 2 as for number 5 & 58.97 & 41.03 \\
\hline
\end{tabular}

\section{CONCLUSION}

In this paper human images segmented from non-human images, innovation of this article allocated to new PC-SVM base human classification, that able to segment human region from non human region. The result of algorithm that earned by testing algorithm in images, confirm the superiority of this proposal algorithm.

\section{REFERENCES}

[1] C. Papageorgiou and T. Poggio. A trainable system for object detection. IJCV, 38(1):15ñ33, 2000.

[2] A. Mohan, C. Papageorgiou, and T. Poggio. Example-based object detection in images by components. PAMI, 23(4):349ñ 361, April 2001.

[3] V. de Poortere, J. Cant, B. Van den Bosch, J. dePrins, F. Fransens, and L. Van Gool. Efficient pedestrian detection: a test case for svm based categorization.

[4] D. M. Gavrila, J. Giebel, and S. Munder. Vision-based pedestrian detection: the protector+ system. Proc. of the IEEE Intelligent Vehicles Symposium, Parma, Italy, 2004.

[5] D. M. Gavrila and V. Philomin. Real-time object detection for smart vehicles. CVPR, Fort Collins, Colorado, USA, pages 87ñ93, 1999.

[6] P. Viola, M. J. Jones, and D. Snow. Detecting pedestrians using patterns of motion and appearance. The 9th ICCV, Nice, France, volume 1, pages 734ñ 741, 2003

[7] R. Ronfard, C. Schmid, and B. Triggs. Learning to parse pictures of people. The 7th ECCV, Copenhagen, Denmark, volume IV, pages 700ก̃714, 2002.

[8] P. Felzenszwalb and D. Huttenlocher. Efficient matching of pictorial structures. CVPR, Hilton Head Island, South Carolina, USA, pages $66 \tilde{7} 75,2000$.

[9] S. Ioffe and D. A. Forsyth. Probabilistic methods for finding people. IJCV, 43(1):45ñ 68, 2001.

[10] K. Mikolajczyk, C. Schmid, and A. Zisserman. Human detection based on a probabilistic assembly of robust part detectors.

[11] K. Mikolajczyk, C. Schmid, and A. Zisserman. Human detection based on a probabilistic assembly of robust part detectors. In Europe Conf. Comp. Vision (ECCV), volume I, pages 69-81, 2004.

[12] Lowe, D.G. (1999) Object recognition from local scale-invariant features.

[13] Lowe, D.G. (1999) Distinctive Image Features from Scale-Invariant Keypoints (2004) International Journal of Computer Vision .

[14] N.Dalal , B.Triggs. Histograms of Oriented Gradients for Human Detection INRIA Rhàone-Alps, 655 avenue de l'Europe, Montbonnot 38334, France

[15] V. Vapnik, The Nature of Statistical Learning Theory. New-York: Springer-Verlag, 1995.

First A. Author: Seyyed Meysam Hosseini was born in Firuzkuh, Iran, in 1984. He received the B.S degree in Electronic engineering from Tarbiat moalem University of Sabzevar, Sabzevar, Iran, in 2007. He is now a M.S student in electrical engineering in University of Birjand, Iran. His research interests include image processing and intelligent computing. E-mail:M.Hosseini@Birjand.ac.ir

B. Author: Hasan Farsi was born in Birjand, Iran, in 1968. He received the B.S. and M.s. degree in electrical engineering from sharif University of Technology, iran respectively, and then he received the $\mathrm{PhD}$ degrees in electrical engineering from University of Surey,UK. He works in Engineering Department as an assistant professor at University of Birjand. E-mail:H.Farsi@Birjand.ac.ir 